\title{
EDITORIAL
}

\section{Physical inactivity: common pathway to peripheral muscle weakness in chronic respiratory diseases?}

\author{
I. Vogiatzis*,\# and S. Zakynthinos*
}

ower limb muscle atrophy and weakness are common in patients with chronic obstructive pulmonary disease (COPD) and make an important contribution to limiting their exercise tolerance [1]. Muscle disuse, hypoxaemia, malnutrition, oxidative stress and systemic inflammation have all been portrayed as possible causes of muscle dysfunction in COPD [2]. As far as systemic inflammation is concerned, the available literature supports cytokine-mediated effects on peripheral muscle atrophy, dysfunction and regeneration [3].

The study by SwALLow et al. [4] in the current issue of the European Respiratory Journal is the first to show that idiopathic scoliosis (a noninflammatory disease) and COPD (currently thought of as a systemic inflammatory disease affecting many tissues and organs in addition to the lungs) share skeletal muscle weakness and wasting as common phenomena. Muscle strength and exercise tolerance were shown to be compromised to a comparable degree in patients with scoliosis and COPD. Interestingly, quadriceps muscle molecular analyses were indicative of peripheral muscle dysfunction manifested in both disease entities by a reduced proportion of Type I myosin heavy-chain isoform (corresponding to Type I oxidative muscle fibres) and by upregulation of local muscle oxidative stress markers. Therefore, the study by SwALLOW et al. [4] presents an interesting paradigm that argues against inflammation as the principal aetiology of peripheral muscle wasting in chronic respiratory diseases and suggests another common aetiological mechanism.

This mechanism was not directly elucidated by SwALLOW et al. [4]. However, this study ultimately points towards muscle deconditioning as a common and important cause of lower limb muscle weakness in both diseases. Indeed, both patient groups exhibited severe and comparable lung function abnormality (i.e. forced expiratory volume in $1 \mathrm{~s}$ of $\sim 30 \%$ predicted) and hypoxaemia, both of which would be expected to exacerbate dyspnoea sensations upon physical exertion. It is conceivable that during the course of the disease, patients with COPD and scoliosis would have adopted a sedentary lifestyle with consequent physical inactivity in order to avoid the occurrence of dyspnoea. This way of living most likely caused

*Dept of Critical Care Medicine and Pulmonary Services, Pulmonary Rehabilitation Centre, Evangelismos Hospital, National \& Kapodistrian University of Athens, and ${ }^{*}$ Dept of Physical Education and Sport Sciences, National \& Kapodistrian University of Athens, Athens, Greece.

CORRESPONDENCE: S. Zakynthinos, National \& Kapodistrian University of Athens, Dept of Critical Care Medicine and Pulmonary Services, Evangelismos Hospital, Athens, Greece. E-mail: szakynthinos@yahoo.com a profound loss of muscle mass as was evidenced by the low fat-free mass index, particularly in scoliosis $\left(14.9 \mathrm{~kg} \cdot \mathrm{m}^{-2}\right)$ [4]. Even in healthy subjects prolonged muscle disuse has been shown to induce a cascade of reactions similar to those seen in COPD and scoliosis, namely altered muscle fibre type distribution, muscle fibre atrophy and oxidative stress $[5,6]$.

An issue of particular importance for the interpretation of the findings of the present study [4] is the relationship between strength data and quadriceps muscle cross-sectional area. Although measures of muscle bulk, e.g., mid-thigh crosssectional area via computed tomography or magnetic resonance imaging, were not performed, the low fat-free mass index found suggests that the quadriceps muscle weakness should be due to a lower quantity of muscle rather than a decrease in muscle performance per unit of muscle volume. Nevertheless, although from the present data it cannot be assumed that weakness is synonymous with atrophy, preliminary additional findings provided by the authors of this study [4] to the Editorial Board showing decreased fibre crosssectional area in quadriceps muscle biopsies (another measure to detect muscle atrophy) from the same patients with scoliosis and COPD, as well as recent data from the same group [7] demonstrating that quadriceps muscle bulk is the primary determinant of strength, support the suggestion that weakness was primarily due to muscle atrophy. The presence of quadriceps muscle atrophy together with the data on myosin heavy-chain shifting (i.e. decrease in Type I and increase in Type II) [4] strengthen the scenario of muscle deconditioning due to physical inactivity as the pivotal factor causing peripheral muscle weakness in both chronic lung diseases.

Another issue is the level of physical activity in the included patients. The authors of the present study [4] presented to the Editorial Board preliminary data in a subset of patients with scoliosis demonstrating substantially reduced physical activity, which is very close to the findings from advanced COPD [8]. As already acknowledged by the authors, the number of patients with COPD and scoliosis studied was relatively small. This is justified by the fact that patients with scoliosis who are naïve to noninvasive ventilation (set as a prerequisite criterion, because it was considered by the authors that noninvasive ventilation, either directly or indirectly by improving overall patient function, might change quadriceps structure and function) are difficult to recruit.

In COPD, reactive oxygen species are elevated during both rest and exercise [9]. The findings by SwALLOW et al. [4] extend those in COPD by showing that oxidative and nitrosative stress 
is also involved in peripheral muscle wasting of the scoliotic patients. Emerging evidence implicates oxidative stress as a key regulator of cell signalling pathways, leading to increased proteolysis and muscle atrophy during periods of prolonged disuse [10]. The mechanism by which inactivity-induced oxidative stress in skeletal muscle contributes to muscle atrophy is currently unknown. However, it appears that oxidative stress could contribute to disuse muscle atrophy by influencing one or more of the following cell signalling pathways: 1) regulation of cytosolic calcium levels and subsequent activation of calpain; 2) control of mitogenactivated protein kinase signalling; and 3) activation of the nuclear factor (NF)- $\kappa$ B pathway [10].

Studies have shown that COPD patients with muscle wasting have increased activity of their protein breakdown pathways, in particular the NF- $\kappa \mathrm{B}$-activated ubiquitin/proteasomal pathway, leading to muscle loss $[11,12]$. As it has recently been recognised that the NF- $\kappa$ B pathway can be activated not only by inflammatory factors (such as tumour necrosis factor- $\alpha$ ) but also by muscle disuse [13], the scientific approach adopted by SwALLOW et al. [4] that is to investigate muscle dysfunction in two distinctively different severe chronic respiratory diseases constitutes a good strategy to distinguish between inflammation-induced and disuse-induced muscle atrophy in chronic lung diseases.

In patients with COPD, pulmonary rehabilitation induces significant adaptations in peripheral muscle fibre size and typology that, in the absence of a decrease in local muscle inflammation, are accompanied by the upregulation of factors governing skeletal muscle hypertrophy and regeneration [14]. Along these lines, future studies should address the question whether patients with severe muscle wasting associated with severe chronic lung disease retain the capacity for muscle remodelling in response to regular exercise training. Despite its explorative nature, the study by SwALLOW et al. [4] suggests that muscle deconditioning due to physical inactivity should be the primary and common determinant of peripheral muscle weakness in chronic lung diseases, inflammatory or noninflammatory, and accordingly states the question of whether rehabilitation should be offered to all patients with chronic respiratory diseases in order to attenuate or even prevent peripheral muscle wasting and weakness. Besides COPD, enhanced systemic inflammatory response is a typical feature of several other chronic diseases, including rheumatoid arthritis and chronic heart failure, all of which share skeletal muscle weakness and wasting as a common phenomenon in the disease course. Therefore, messages taken from the present study [4] might also be applicable in all these diseases, regardless of different primary organ pathology.

\section{STATEMENT OF INTEREST}

None declared.

\section{REFERENCES}

1 Debigare $\mathrm{R}$, Cote $\mathrm{CH}$, Maltais F. Peripheral muscle wasting in chronic obstructive pulmonary disease. Am J Respir Crit Care Med 2001; 164: 1712-1717.

2 Agustí A. Systemic effects of chronic obstructive pulmonary disease. Proc Am Thorac Soc 2005; 2: 367-370.

3 Langen R, Schols A. Inflammation: friend or foe of muscle remodeling in COPD? Eur Respir J 2007; 30: 605-607.

4 Swallow EB, Barreiro E, Gosker H, et al. Quadriceps muscle strength in scoliosis. Eur Respir J 2009; 34: 1429-1435.

5 Kondo H, Miura M, Itokawa Y. Oxidative stress in skeletal muscle atrophied by immobilization. Acta Physiol Scand 1991; 142: 527-528.

6 Boonyarom O, Inui K. Atrophy and hypertrophy of skeletal muscles: structural and functional aspects. Acta Physiologica 1006; 188: 77-89.

7 Seymour JM, Ward K, Sidhu PS, et al. Ultrasound measurement of rectus femoris cross-sectional area and the relationship with quadriceps strength in COPD. Thorax 2009; 64: 418-423.

8 Pitta F, Troosters T, Spruit MA, et al. Characteristics of physical activities in daily life in chronic obstructive pulmonary disease. Am J Respir Crit Care Med 2005; 171: 972-977.

9 Koechlin C, Couillard A, Simar D, et al. Does oxidative stress alter quadriceps endurance in chronic obstructive pulmonary disease? Am J Respir Crit Care Med 2004; 169: 1022-1027.

10 Powers SK, Kavazis AN, McClung JM. Oxidative stress and disuse muscle atrophy. J Appl Physiol 2007; 102: 2389-2397.

11 Agusti A, Morla M, Sauleda J, et al. NF-кB activation and iNOS upregulation in skeletal muscle of patients with COPD and low body weight. Thorax 2004; 59: 483-487.

12 Rutten E, Franssen F, Engelen M, et al. Greater whole-body myofibrillar protein breakdown in cachectic patients with chronic obstructive pulmonary disease. Am J Clin Nutr 2006; 83: 829-834.

13 Jackman RW, Kandarian SC. The molecular basis of skeletal muscle atrophy. Am J Physiol Cell Physiol 2004; 287: C834-C843.

14 Vogiatzis I, Stratakos G, Simoes D, et al. Effects of rehabilitative exercise on peripheral muscle TNF $\alpha$, IL-6, IGF-I and MyoD expression in patients with COPD. Thorax 2007; 62: 950-956. 